\title{
Fault-free Hamiltonian cycles in crossed cubes with conditional link faults
}

\author{
Hao-Shun Hung a , Jung-Sheng Fu ${ }^{b}$, Gen-Huey Chen ${ }^{\mathrm{a}, *}$ \\ a Department of Computer Science and Information Engineering, National Taiwan University, Taipei 10764, Taiwan \\ ${ }^{\mathrm{b}}$ Department of Electronics Engineering, National United University, Miaoli, Taiwan
}

Received 16 August 2006; received in revised form 23 April 2007; accepted 19 May 2007

\begin{abstract}
The crossed cube, which is a variation of the hypercube, possesses some properties superior to the hypercube. In this paper, assuming that each node is incident with at least two fault-free links, we show that an $n$-dimensional crossed cube contains a fault-free Hamiltonian cycle, even if there are up to $2 n-5$ link faults. The result is optimal with respect to the number of link faults tolerated. We also verify that the assumption is practically meaningful by evaluating its occurrence probability, which is very close to 1 .
\end{abstract}

(C) 2007 Elsevier Inc. All rights reserved.

Keywords: Conditional link fault; Crossed cube; Fault-tolerant embedding; Forbidden faulty set model; Hamiltonian cycle; Hypercube; Interconnection network

\section{Introduction}

The hypercube is a popular interconnection network (network for short) with many attractive properties such as regularity, symmetry, small diameter, strong connectivity, recursive construction, partition ability, and relatively low link complexity [25]. On the other hand, the crossed cube [6,7], which can result by changing some connections of the hypercube, is superior to the hypercube in diameter and mean distance. The diameter of an $n$-dimensional crossed cube is $\lceil(n+1) / 2\rceil$, which is about one half of the diameter of an $n$-dimensional hypercube. The mean distance of the crossed cube is smaller than the hypercube.

The crossed cube has been studied extensively in the literature $[3,4,8,11-13,17,19,20,29]$. It was demonstrated in [19] that a $\left(2^{n}-1\right)$-node complete binary tree can be embedded into a $2^{n}$-node crossed cube with dilation one. The dilation will go up to two if the same tree is embedded into a $2^{n}$-node hypercube (see [28]). Path embedding in the crossed cube can be found in $[11,12]$. The connectivity of an $n$-dimensional crossed cube is $n$ (see [20]). It was shown in [3] that both the $n$-wide diameter and the ( $n-1)$-fault diameter of an $n$-dimensional crossed cube were $\lceil n / 2\rceil+2$. In [29], the crossed cube was shown to be pancyclic. It was

\footnotetext{
* Corresponding author. Tel.: +8862 23625336x427; fax: +886223628167.

E-mail address: ghchen@csie.ntu.edu.tw (G.-H. Chen).
} 
further shown in $[17,29]$ that an $n$-dimensional crossed cube is $(n-2)$-Hamiltonian, $(n-3)$-Hamiltonian-connected, and $(n-2)$-fault-tolerant pancyclic. Recently, fault diagnosis of the crossed cube was explored in $[4,13]$.

The random fault model, which was considered in [15,17,29,30], assumed that faults might happen anywhere in a network without any restriction. On the other hand, Harary introduced in [16] the concept of conditional faults. Let $P$ represent a property of a graph $G$ and $S$ be a vertex subset of $G$. The $P$-connectivity of $G$ was defined to be the minimum $|S|$ so that $G-S$ is disconnected and every component of $G-S$ satisfies the property $P$. Considering $P$ the property that each node is incident with at least one fault-free node, $P$-connectivities were computed for hypercubes [9], $k$-ary $n$-cubes [5], cubes-connected cycles [23], undirected de Bruijn networks [23], and Kautz networks [23]. In addition, under the same assumption (i.e., the property $P$ ), the diameters of hypercubes and star graphs were computed in [21,24], respectively.

In the past 20 years, few Hamiltonian properties were derived on networks with conditional faults, although $P$-connectivities were obtained for some networks. It appears that Hamiltonicity problems on networks become more difficult, when conditional faults are assumed. Previous results were obtained only on hypercubes and $k$-ary $n$-cubes. Under the assumption that each node is incident with two or more fault-free links, it was shown in $[1,2]$ that a $k$-ary $n$-dimensional hypercube (an $n$-dimensional hypercube) contains a fault-free Hamiltonian cycle, even if there are up to $4 n-5(2 n-5)$ link faults. Besides, it was shown in [26] that an $n$-dimensional hypercube with $2 n-5$ link faults is strongly (fault-free) Hamiltonian laceable.

In this paper, with the same assumption as [1,2], we show that an $n$-dimensional crossed cube contains a fault-free Hamiltonian cycle, even if there are up to $2 n-5$ link faults. The result is optimal with respect to the number of link faults tolerated. The rest of this paper is organized as follows. In Section 2, the structure of the crossed cube is reviewed. Some necessary definitions and notations are also introduced. In Section 3, some favorable properties of the crossed cube are derived. These properties will help the derivation of the main result. In Section 4, the main result and its optimality are shown. In Section 5, the probability that the assumption holds when there are $2 n-5$ link faults is analyzed. In Section 6, this paper concludes with some remarks.

\section{Preliminaries}

A network is conveniently represented with an undirected graph, where the vertices (edges) of the graph denote the nodes (links) of the network. Throughout this paper, vertex and node, edge and link, and graph and network are used interchangeably. Moreover, we use $C Q_{n}$ to denote an $n$-dimension crossed cube. $C Q_{n}$ can be constructed recursively. Initially, $C Q_{1}$ contains a link whose two end nodes are labeled with 0 and 1, respectively, and $C Q_{2}$ is isomorphic to a two-dimensional hypercube. For $n \geqslant 3, C Q_{n}$ can be obtained by joining two $C Q_{n-1}$ 's, denoted by $C Q_{n-1}^{0}$ and $C Q_{n-1}^{1}$, with $2^{n-1}$ links, as described below.

Each node label of $C Q_{n-1}^{0}\left(C Q_{n-1}^{1}\right)$ is preceded with a bit 0 (1). There is a link joining a node $u=0 u_{n-2} u_{n-3} \ldots u_{0}$ in $C Q_{n-1}^{0}$ with another node $v=1 v_{n-2} v_{n-3} \ldots v_{0}$ in $C Q_{n-1}^{1}$ if and only if $\left(u_{2 i+1} u_{2 i}, v_{2 i+1} v_{2 i}\right) \in\{(00,00),(10,10),(01,11),(11,01)\}$ for all $0 \leqslant i \leqslant\lfloor(n-1) / 2\rfloor-1$ and $u_{n-2}=v_{n-2}$ if $n$ is even. In subsequent discussion $(u, v)$ is referred to as a crossing link of $C Q_{n}$ and $u(v)$ is referred to as the crossing node of $v(u)$ with respect to $C Q_{n}$. For convenience, we use $c_{n}(v)$ to denote the crossing node of $v$ with respect to $C Q_{n}$. So, we have $c_{n}(v)=u$ and $c_{n}(u)=v$. Fig. 1 depicts $C Q_{3}$ and $C Q_{4}$, where $(000,100),(010,110)$, $(001,111)$ and $(011,101)$ are four crossing links of $C Q_{3}$.

Formally, $C Q_{n}$ can be defined as follows, where we use $u_{2 i+1} u_{2 i} \sim v_{2 i+1} v_{2 i}$ to denote $\left(u_{2 i+1} u_{2 i}, v_{2 i+1} v_{2 i}\right) \in$ $\{(00,00),(10,10),(01,11),(11,01)\}$.

Definition 1 [6]. The node set of $C Q_{n}$ is $\left\{v_{n-1} v_{n-2} \ldots v_{0} \mid v_{i} \in\{0,1\}\right.$ for all $\left.0 \leqslant i \leqslant n-1\right\}$. Two nodes $u=u_{n-1} u_{n-2} \ldots u_{0}$ and $v=v_{n-1} v_{n-2} \ldots v_{0}$ of $C Q_{n}$ are adjacent if and only if there exists $0 \leqslant d \leqslant n-1$ so that the following four conditions are satisfied:

(1) $u_{n-1} u_{n-2} \ldots u_{d+1}=v_{n-1} v_{n-2} \ldots v_{d+1}$, if $d<n-1$;

(2) $u_{d}=\overline{v_{d}}\left(\overline{v_{d}}\right.$ is the complement of $\left.v_{d}\right)$;

(3) $u_{d-1}=v_{d-1}$, if $d$ is odd;

(4) $u_{2 i+1} u_{2 i} \sim v_{2 i+1} v_{2 i}$ for all $0 \leqslant i \leqslant\lfloor d / 2\rfloor-1$, if $d \geqslant 2$. 

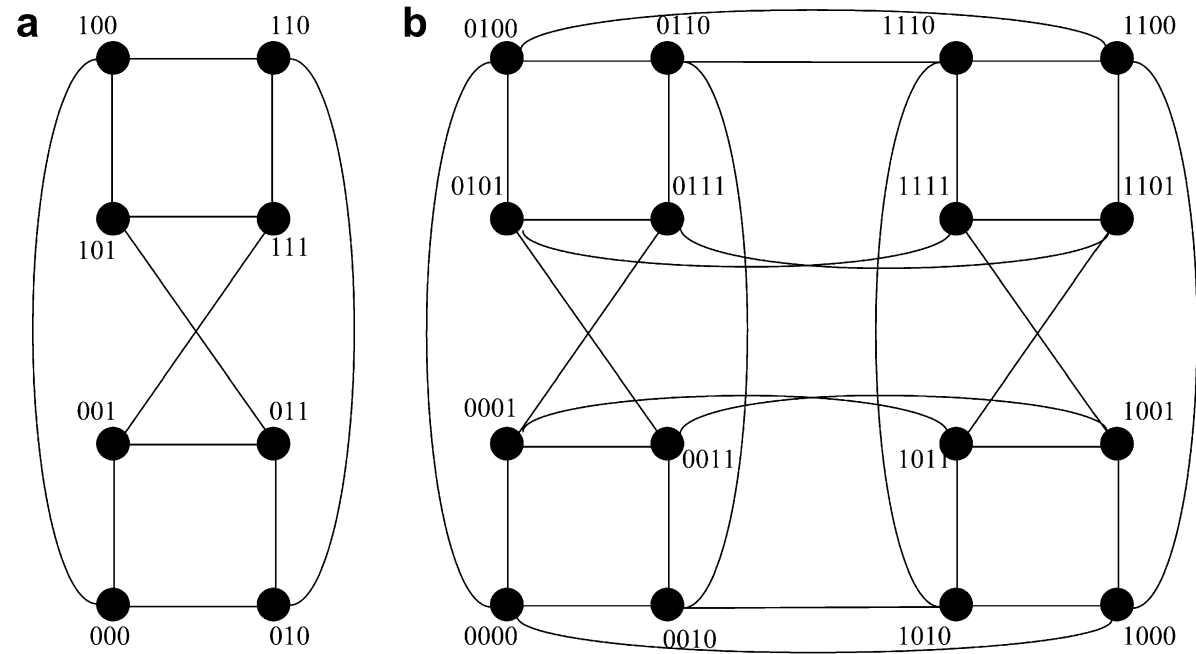

Fig. 1. $C Q_{n}$. (a) $n=3$. (b) $n=4$.

Notice that $(u, v)$ above is a crossing link of $C Q_{d+1}$, which joins $C Q_{d}^{0}$ with $C Q_{d}^{1}$. Such a link is called a $d$-link. Every node in $C Q_{n}$ is incident with $n$ links, which are 0 -link, 1-link, 2-link, .., $(n-1)$-link. A path (cycle) in a graph $G$ is called a Hamiltonian path (cycle) if it contains every vertex of $G$ exactly once. The following two lemmas were shown in [17].

Lemma 1 [17]. $A C Q_{n}$ with at most $n-2$ link faults contains a fault-free Hamiltonian cycle, where $n \geqslant 3$.

Lemma 2 [17]. Suppose that there are $f_{e}$ link faults and $f_{v}$ node faults in a $C Q_{n}$. If $f_{e}+f_{v} \leqslant n-3$, then for every two fault-free nodes $u, v$ of the $C Q_{n}$, there is a fault-free path of length $2^{n}-f_{v}-1$ joining $u$ and $v$ in the $C Q_{n}$, where $n \geqslant 3$.

In fact, the fault-free path of length $2^{n}-f_{v}-1$ in Lemma 2 is a Hamiltonian path in the graph that results by removing the $f_{v}$ faulty nodes from the $C Q_{n}$.

\section{Properties}

In this section, some properties of the crossed cube are derived. They will be used when we construct a faultfree Hamiltonian cycle in a faulty crossed cube, which was described in the next section.

Lemma 3 [20]. Suppose that $(u, v)$ is a d-link in $C Q_{n}$, where $d$ is odd or $d=n-2$. Then, $\left(c_{n}(u), c_{n}(v)\right)$ is also a d-link.

For a graph $G$, we use $V(G)(E(G))$ to denote the vertex set (edge set) of $G$. Also we use $P_{x, y}$ to denote a path between two nodes $x, y$ of the crossed cube.

Lemma 4. Suppose that $u, v, x$ and $y$ are four distinct nodes in $C Q_{n}$, where $n \geqslant 4$. There exist $P_{u, v}$ and $P_{x, y}$ so that we have $V\left(P_{u, v}\right) \cap V\left(P_{x, y}\right)=\varnothing$ and $V\left(P_{u, v}\right) \cup V\left(P_{x, y}\right)=V\left(C Q_{n}\right)$.

Proof. We prove this lemma by induction on $n$. For $n=4$, the lemma can be easily verified by exhaustive search (by the aid of a computer program) [33]. We assume that the lemma holds for $n=k \geqslant 4$. The situation of $n=k+1$ is discussed with the following four cases.

Case 1: $u$ is in $V\left(C Q_{k}^{0}\right)$ and $x$ is in $V\left(C Q_{k}^{1}\right)$. If $v$ is in $V\left(C Q_{k}^{0}\right)$ and $y$ is in $V\left(C Q_{k}^{1}\right)$, then by Lemma 2, there are a Hamiltonian path between $u$ and $v$ in $C Q_{k}^{0}$ and a Hamiltonian path between $x$ and $y$ in $C Q_{k}^{1}$, which are a desired $P_{u, v}$ and a desired $P_{x, y}$, respectively. 
If $v$ is in $V\left(C Q_{k}^{1}\right)$ and $y$ is in $V\left(C Q_{k}^{0}\right)$, then there is a Hamiltonian path between $u$ and $y$ in $C Q_{k}^{0}$ similarly. A link $(s, t)$ in the path can be found so that we have $c_{k+1}(s), c_{k+1}(t) \notin\{x, v\}$. Refer to Fig. 2a, where $s^{\prime}=c_{k+1}(s)$ and $t^{\prime}=c_{k+1}(t)$. By the induction hypothesis, there are $P_{s^{\prime}, v}$ and $P_{x, t^{\prime}}$ so that we have $V\left(P_{s^{\prime}, v}\right) \cap V\left(P_{x, t^{\prime}}\right)=\varnothing$ and $V\left(P_{s^{\prime}, v}\right) \cup V\left(P_{x, t^{\prime}}\right)=V\left(C Q_{k}^{1}\right)$. Hence a desired $P_{u, v}$ and a desired $P_{x, y}$ can be constructed as the two bold paths in Fig. 2a.

If $v$ and $y$ are in $V\left(C Q_{k}^{1}\right)$, then we select a node $s$ in $C Q_{k}^{0}$ so that we have $s \notin\{u, v, y\}$ and $c_{k+1}(s) \neq x$. A desired $P_{u, v}$ and a desired $P_{x, y}$ can be constructed as shown in Fig. 2b, where we have $s^{\prime}=c_{k+1}(s), \quad V\left(P_{u, v}\right) \cap V\left(P_{y, s}\right)=\varnothing, \quad V\left(P_{u, v}\right) \cup V\left(P_{y}, s\right)=V\left(C Q_{k}^{0}\right)$, and $V\left(P_{x, s^{\prime}}\right)=V\left(C Q_{k}^{1}\right)$ (i.e., $P_{x, s^{\prime}}$ is a Hamiltonian path in $\left.C Q_{k}^{1}\right)$. The discussion for the situation that $v$ and $y$ are in $V\left(C Q_{k}^{1}\right)$ is similar.

Case 2: $u$ is in $V\left(C Q_{k}^{1}\right)$ and $x$ is in $V\left(C Q_{k}^{1}\right)$. The construction of a desired $P_{u, v}$ and a desired $P_{x, y}$ is similar to Case 1.

Case 3: $u$ and $x$ are in $V\left(C Q_{k}^{0}\right)$. If $v$ is in $V\left(C Q_{k}^{0}\right)$ and $y$ is in $V\left(C Q_{k}^{1}\right)$ or $v$ is in $V\left(C Q_{k}^{1}\right)$ and $y$ is in $V\left(C Q_{k}^{0}\right)$, then a desired $P_{u, v}$ and a desired $P_{x, y}$ can be obtained by the construction method of Fig. $2 \mathrm{~b}$.

If $v$ and $y$ are in $V\left(C Q_{k}^{0}\right)$, then we arbitrarily select a link $(s, t)$ in $P_{u, v}$. A desired $P_{u, v}$ and a desired $P_{x, y}$ can be constructed as shown in Fig. 3a, where we have $s^{\prime}=c_{k+1}(s), t^{\prime}=c_{k+1}(t), V\left(P_{u, v}\right) \cap V\left(P_{x, y}\right)=$ $\varnothing, \quad V\left(P_{u, v}\right) \cup V\left(P_{x, y}\right)=V\left(C Q_{k}^{0}\right)$, and $V\left(P_{s^{\prime}, t^{\prime}}\right)=V\left(C Q_{k}^{1}\right)$.

If $v$ and $y$ are in $V\left(C Q_{k}^{1}\right)$, then we arbitrarily select nodes $s, t$ from $V\left(C Q_{k}^{0}\right)-\{x, u\}$ so that we have $c_{k+1}(s)$, $c_{k+1}(t) \notin\{v, y\}$. A desired $P_{u, v}$ and a desired $P_{x, y}$ can be constructed as shown in Fig. 3b, where we have $s^{\prime}=c_{k+1}(s), t^{\prime}=c_{k+1}(t), \quad V\left(P_{u, s}\right) \cap V\left(P_{x, t}\right)=\varnothing, \quad V\left(P_{u, s}\right) \cup V\left(P_{x, t}\right)=V\left(C Q_{k}^{0}\right), \quad V\left(P_{y, t^{\prime}}\right) \cap V\left(P_{v, s^{\prime}}\right)=\varnothing$, and $\left(P_{y, t^{\prime}}\right) \cup V\left(P_{v, s^{\prime}}\right)=V\left(C Q_{k}^{1}\right)$.

Case 4: $u$ and $x$ are in $V\left(C Q_{k}^{1}\right)$. The construction of a desired $P_{u, v}$ and a desired $P_{x, y}$ is similar to Case 3.

Lemma 5. Suppose that $(u, v)$ is an arbitrary fault-free $(n-1)$-link in a $C Q_{n}$ with at most $n-2$ link faults, where $n \geqslant 4$. There exists a fault-free Hamiltonian cycle in the $C Q_{n}$ that contains $(u, v)$.

Proof. Let $f_{c}$ be the number of faulty crossing links of the $C Q_{n}$ and $f_{0}\left(f_{1}\right)$ be the number of link faults in $\left(C Q_{n-1}^{0}\right)\left(C Q_{n-1}^{1}\right)$. We have $f_{c}+f_{0}+f_{1} \leqslant n-2$. Without loss of generality, we assume $f_{0} \geqslant f_{1}$. We also assume that $u$ is in $V\left(C Q_{n-1}^{0}\right)$ and $v$ is in $V\left(C Q_{n-1}^{1}\right)$. For $n=4$, the lemma can be easily verified by exhaustive search. For $n \geqslant 5$, three cases are discussed below:

Case 1: $f_{0}<n-3$. There is a fault-free $(n-1)$-link $\left(x, x^{\prime}\right) \neq(u, v)$, where $x$ is in $V\left(C Q_{n-1}^{0}\right)$ and $x^{\prime}=c_{n}(x)$. By Lemma 2, there are a fault-free Hamiltonian path between $u$ and $x$ in and a fault-free Hamiltonian path
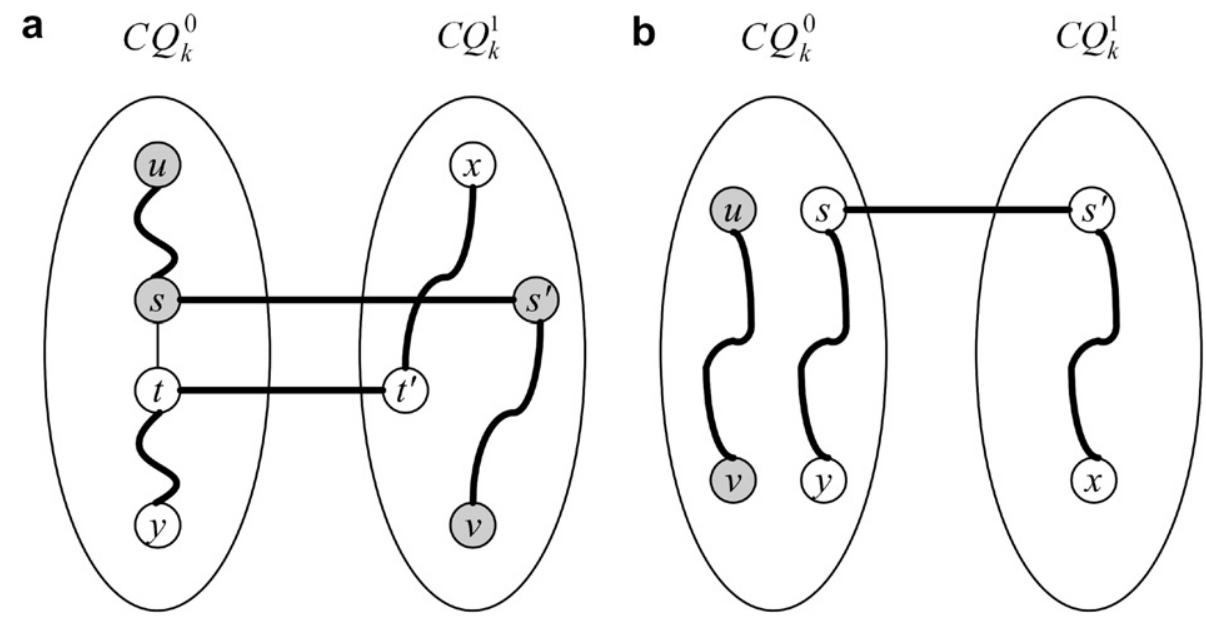

Fig. 2. Construction of $P_{u, v}$ and $P_{x, y}$ when $u$ is in $V\left(C Q_{k}^{0}\right)$ and $x$ is in $V\left(C Q_{k}^{1}\right)$. (a) $v$ is in $V\left(C Q_{k}^{0}\right)$ and $y$ is in $V\left(C Q_{k}^{1}\right)$. (b) $v$ and $y$ are in $V\left(C Q_{k}^{0}\right)$. 
a

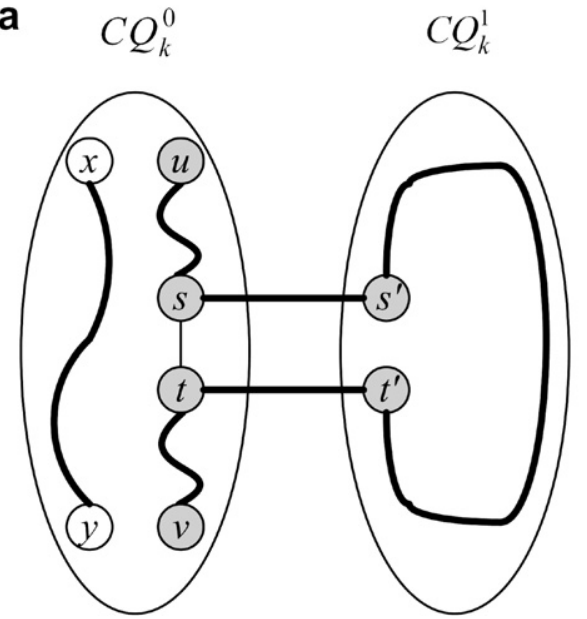

b $\quad C Q_{k}^{0} \quad C Q_{k}^{1}$

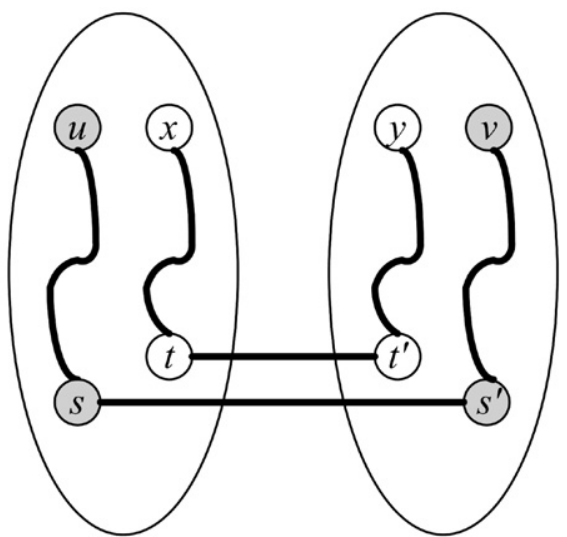

Fig. 3. Construction of $P_{u, v}$ and $P_{x, y}$ when $u$ and $x$ are in $V\left(C Q_{k}^{0}\right)$. (a) $v$ and $y$ are in $V\left(C Q_{k}^{0}\right)$. (b) $v$ and $y$ are in $V\left(C Q_{k}^{1}\right)$.

a
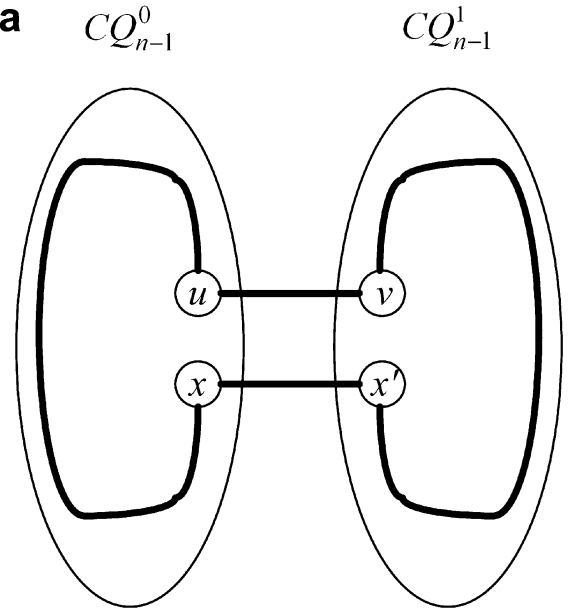

b $\quad C Q_{n-1}^{0}$

$C Q_{n-1}^{1}$

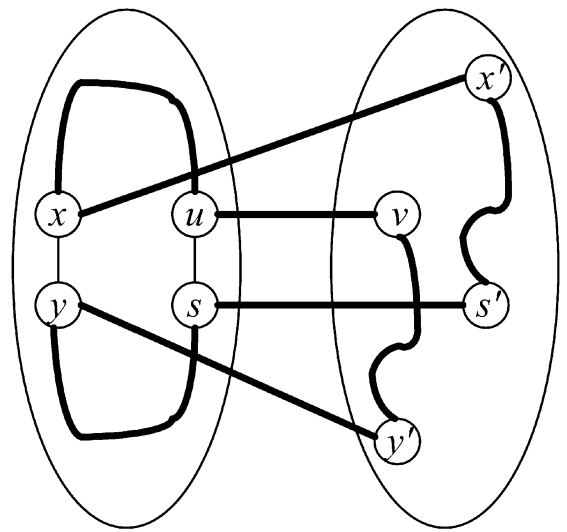

Fig. 4. Construction of a fault-free Hamiltonian cycle in $C Q_{n}$ that contains $(u, v)$. (a) $f_{0}<n-3$. (b) $f_{0}=n-2$.

between $u$ and $x^{\prime}$ in $C Q_{n-1}^{0}$. A desired fault-free Hamiltonian cycle can be constructed as the bold cycle in Fig. 4a.

Case 2: $f_{0}=n-3$. By Lemma 1, there is a fault-free Hamiltonian cycle in $C Q_{n-1}^{0}$. Since $f_{0}+f_{1}+f_{c} \leqslant n-2$, we have $f_{c} \leqslant 1$. There is a fault-free $(n-1)$-link $\left(x, x^{\prime}\right) \neq(u, v)$, where $x$ is neighboring to $u$ in the Hamiltonian cycle and $x^{\prime}=c_{n}(x)$. By Lemma 2, there is a fault-free Hamiltonian path between $x^{\prime}$ and $v$ in $C Q_{n-1}^{1}$. A desired fault-free Hamiltonian cycle can be obtained by the construction method of Fig. 4a.

Case 3: $f_{0}=n-2$. We have $f_{c}=f_{1}=0$. Suppose that $(x, y)$ is a faulty link in $C Q_{n-1}^{0}$. There are $n-3$ link faults in $C Q_{n-1}^{0}$, exclusive of $(x, y)$. By Lemma 1 (imagining that $(x, y)$ is fault-free), there exists a Hamiltonian cycle in that can avoid the $n-3$ link faults. If $(x, y)$ is not contained in the Hamiltonian cycle, then we select a fault-free $(n-1)$-link $\left(t, t^{\prime}\right) \neq(u, v)$, where $t$ is neighboring to $u$ in the Hamiltonian cycle and $t^{\prime}=c_{n}(t)$. A desired fault-free Hamiltonian cycle can be obtained by the construction method of Fig. 4a. Otherwise $((x, y)$ is contained in the Hamiltonian cycle), if $x=u$, then a desired fault-free Hamiltonian cycle can be obtained by the construction method of Fig. 4a (replacing $\left(x, x^{\prime}\right)$ with $\left(y, y^{\prime}\right)$, where $\left.y^{\prime}=c_{n}(y)\right)$. If $x \neq u$, then a desired fault-free Hamiltonian cycle can be constructed as shown in Fig. 4b, where $s \notin\{x, y\}$ 
is neighboring to $u$ in the Hamiltonian cycle of $C Q_{n-1}^{0}$ and $\left(x, x^{\prime}\right),\left(y, y^{\prime}\right),\left(s, s^{\prime}\right)$ are fault-free $(n-1)$ links.

Lemma 6. Suppose that $(u, v)$ is an arbitrary fault-free $d$-link in a $C Q_{n}$ with at most $n-2$ link faults, where $1 \leqslant d \leqslant n-2$ is odd and $n \geqslant 4$. There exists a fault-free Hamiltonian cycle in the $C Q_{n}$ that contains $(u, v)$.

Proof. We prove this lemma by induction on $n$. For $n=4$, the lemma can be easily verified by exhaustive search. We assume that the lemma holds for $n=k \geqslant 4$. Then we consider the situation of $n=k+1$. Let $f_{c}$ be the number of faulty crossing links of the $C Q_{k+1}$ and $f_{0}\left(f_{1}\right)$ be the number of link faults in $C Q_{k}^{0}\left(C Q_{k}^{1}\right)$. We have $f_{0}+f_{1}+f_{c} \leqslant k-1$. Without loss of generality, we assume $f_{0} \geqslant f_{1}$. Two cases are discussed below.

Case 1: $f_{0} \leqslant k-2$. If $(u, v)$ is in $E\left(C Q_{k}^{0}\right)$, then by the induction hypothesis, there exists a fault-free Hamiltonian cycle in $C Q_{k}^{0}$ that contains $(u, v)$. Moreover, there exists a link $(s, t) \neq(u, v)$ in the Hamiltonian cycle so that $\left(s, s^{\prime}\right)$ and $\left(t, t^{\prime}\right)$ are fault-free, where $s^{\prime}=c_{k+1}(s)$ and $t^{\prime}=c_{k+1}(t)$. Since there are $2^{k}(>2(k-1)+1)$ links contained in the Hamiltonian cycle, such a link $(s, t)$ exists. On the other hand, we have $f_{1} \leqslant\lfloor(k-1) /$ $2\rfloor<k-3$, as a consequence of $f_{0} \geqslant f_{1}$ and $f_{0}+f_{1}+f_{c} \leqslant k-1$. Then, by Lemma 2, there is a fault-free Hamiltonian path between $s^{\prime}$ and $t^{\prime}$ in $C Q_{k}^{1}$. A desired fault-free Hamiltonian cycle can be constructed as shown in Fig. 5a. The discussion for the situation that $(u, v)$ is in $E\left(C Q_{k}^{1}\right)$ is similar.

Case 2: $f_{0}=k-1$. We have $f_{c}=f_{1}=0$. Suppose that $(x, y)$ is a faulty link in $C Q_{k}^{0}$ such that we have $\left\{x^{\prime}, y^{\prime}\right\} \neq\{u, v\}$, where $x^{\prime}=c_{k+1}(x)$ and $y^{\prime}=c_{k+1}(y)$. There are $k-2$ link faults in $C Q_{k}^{0}$, exclusive of $(x, y)$. We first assume $(u, v)$ is in $E\left(C Q_{k}^{0}\right)$. By the induction hypothesis (imagining that $(x, y)$ is fault-free) there exists a Hamiltonian cycle, denoted by $C$, in $C Q_{k}^{0}$ that contains $(u, v)$, but does not contain the $k-2$ link faults. A desired fault-free Hamiltonian cycle can be obtained by the construction method of Fig. 5a (replacing $(s, t)$ with $(x, y)$ if $(x, y)$ is contained in $C$, and replacing $(s, t)$ with another link in $C$ if $(x, y)$ is not contained in $C)$.

Then we assume that $(u, v)$ is in $E\left(C Q_{k}^{1}\right)$. By Lemma 1 (imagining that $(x, y)$ is fault-free), there exists a Hamiltonian cycle, denoted by $C^{\prime}$, in $C Q_{k}^{0}$ that can avoid the $k-2$ link faults. If $(x, y)$ is not contained in $C^{\prime}$, then we select a link $(s, t)$ from $C^{\prime}$ so that we have $\left\{s^{\prime}, t^{\prime}\right\} \cap\{u, v\}=\varnothing$, where $s^{\prime} c_{k+1}(s)$ and $t^{\prime} c_{k+1}(t)$. By Lemma 4 , there are two paths $P_{u, s^{\prime}}$ and $P_{v, t^{\prime}}$ that satisfy $V\left(P_{u, s^{\prime}}\right) \cap V\left(P_{v, t^{\prime}}\right)=\varnothing$ and $V\left(P_{u, s^{\prime}}\right) \cup$ $V\left(P_{v, t^{\prime}}\right)=V\left(C Q_{k}^{1}\right)$. Besides, they are fault-free because $f_{1}=0$. A desired fault-free Hamiltonian cycle can be constructed as shown in Fig. 5 b.

If $(x, y)$ is contained in $C^{\prime}$ and $\left\{x^{\prime}, y^{\prime}\right\} \cap\{u, v\}=\varnothing$, then a desired fault-free Hamiltonian cycle can be obtained by the construction method of Fig. 5b (replacing $(s, t)$ with $(x, y)$ ). If $(x, y)$ is contained in $C^{\prime}$ and $\left\{x^{\prime}, y^{\prime}\right\} \cap\{u, v\}=\varnothing$, then a desired fault-free Hamiltonian cycle can be obtained as shown in Fig. 5c. In Fig. 5c, $x^{\prime}=u$ is assumed, and the fault-free path between $v$ and $y^{\prime}$ that contains all nodes in $V\left(C Q_{k}^{1}\right)-\{u\}$ can be assured by Lemma 2 (imagining that $u$ is faulty).

In the next section, we show the main result of this paper, which is stated below.
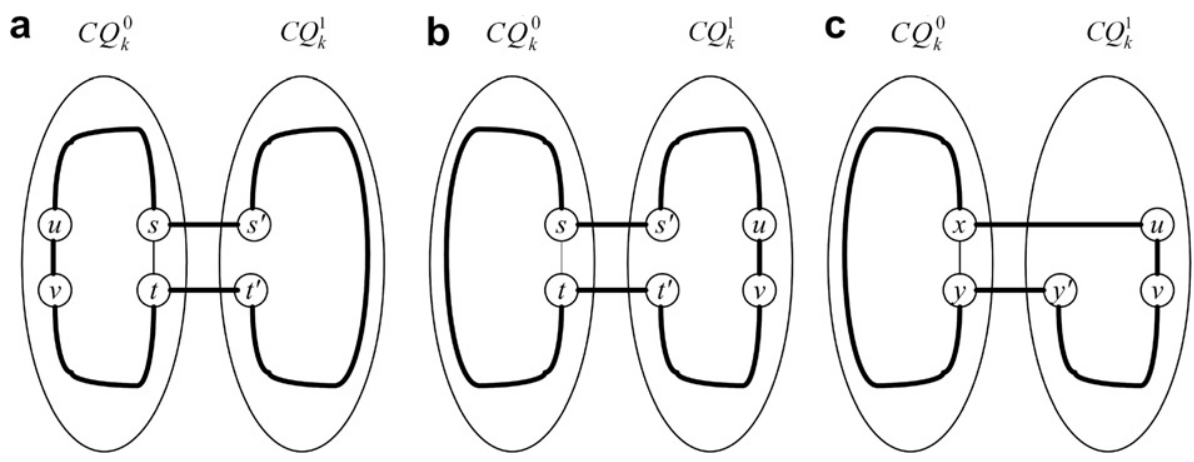

Fig. 5. Construction of a fault-free Hamiltonian cycle in $C Q_{k+1}$ that contains $(u, v)$. (a) $f_{0} \leqslant k-2$. (b) $(x, y)$ is not contained in $C^{\prime}$. (c) $(x, y)$ is contained in $C^{\prime}$ and $\left\{x^{\prime}, y^{\prime}\right\} \cap\{u, v\} \neq \varnothing$. 
Theorem 1. With the assumption that each node is incident with at least two fault-free links, there is a fault-free Hamilton cycle in a $C Q_{n}$ with up to $2 n-5$ link faults, where $n \geqslant 3$. Moreover, the result is optimal with the respect to the number of link faults tolerated.

\section{Proof of Theorem 1}

For $n=3$, the correctness of the theorem can be assured by Lemma 1. So we assume $n \geqslant 4$. We prove the theorem by induction. When $n=4$, the theorem can be easily verified by exhaustive search. We assume that the theorem holds for $n=k \geqslant 4$. In the rest of this section, the situation of $n=k+1$ is considered.

Let $f_{c}$ be the number of faulty crossing links of the $C Q_{k+1}$ and $f_{0}\left(f_{1}\right)$ be the number of link faults in $C Q_{k}^{0}\left(C Q_{k}^{1}\right)$. We have $f_{0}+f_{1}+f_{c} \leqslant 2 k-3$. Without loss of generality, we assume $f_{0} \geqslant f_{1}$. Three cases are discussed below:

Case 1: $f_{0} \leqslant 2 k-5$. There is at most one node in $C Q_{k}^{0}$ that is incident with only one fault-free link in $C Q_{k}^{0}$ (the other fault-free link is a crossing link of the $C Q_{k+1}$ ), for otherwise there are at least $2 k-3$ link faults in $C Q_{k}^{0}$, which is a contradiction.

We first assume that every node in $C Q_{k}^{0}$ is incident with at least two fault-free links in $C Q_{k}^{0}$. By the induction hypothesis, there exists a fault-free Hamiltonian cycle in $C Q_{k}^{0}$. A link $(s, t)$ can be found in the Hamiltonian cycle so that $\left(s, s^{\prime}\right)$ and $\left(t, t^{\prime}\right)$ are fault-free, where $s^{\prime}=c_{k+1}(s)$ and $t^{\prime}=c_{k+1}(t)$. In addition, $(s, t)$ should be selected to be a $(k-1)$-link if $f_{1}=k-2$ (it surely exists because $f_{c} \leqslant 1$ as $f_{1}=k-2$ ). If $f_{1} \leqslant k-3$, then by Lemma 2 there is a fault-free Hamiltonian path between $s^{\prime}$ and $t^{\prime}$ in $C Q_{k}^{1}$. If $f_{1}=k-2$, then by Lemma 3 $\left(s^{\prime}, t^{\prime}\right)$ is a $(k-1)$-link, and by Lemma 5 there is a fault-free Hamiltonian cycle in $C Q_{k}^{1}$ that contains $\left(s^{\prime}, t^{\prime}\right)$. A desired fault-free Hamiltonian cycle can be constructed as shown in Fig. 6a.

Then we assume that a node $p$ in $C Q_{k}^{0}$ is incident with only one fault-free link in $C Q_{k}^{0}$. Hence, we have $f_{0} \geqslant k-1$ (thus $\left.f_{1}+f_{c} \leqslant k-2\right)$ and $\left(p, p^{\prime}\right)$ is fault-free, where $p^{\prime}=c_{k+1}(p)$. A faulty link $(p, q)$ with $\left(q, q^{\prime}\right)$ fault-free can be found in $C Q_{k}^{0}$, where $q^{\prime}=c_{k+1}(q)$. In addition, $(p, q)$ should be selected to be a $d$-link for some odd $d$ if $f_{1}=k-2$ (thus $f_{c}=0$ ). By the induction hypothesis (imagining that $(p, q)$ is fault-free because $p$ is incident with only one fault-free link in $C Q_{k}^{0}$ ), there is a Hamiltonian cycle that can avoid all faulty links but $(p, q)$ in $C Q_{k}^{0}$. Notice that $(p, q)$ should be included in the Hamiltonian cycle.

If $f_{1} \leqslant k-3$, then by Lemma 2 there exists a fault-free Hamiltonian path between $p^{\prime}$ and $q^{\prime}$ in $C Q_{k}^{1}$. If $f_{1}=k-2$, then by Lemma $3\left(p^{\prime}, q^{\prime}\right)$ is a $d$-link, and by Lemma 6 there exists a fault-free Hamiltonian cycle in $C Q_{k}^{1}$ that contains $\left(p^{\prime}, q^{\prime}\right)$. A desired fault-free Hamiltonian cycle can be obtained by the construction method of Fig. 6a (replacing $(s, t)$ with $(p, q))$.

a

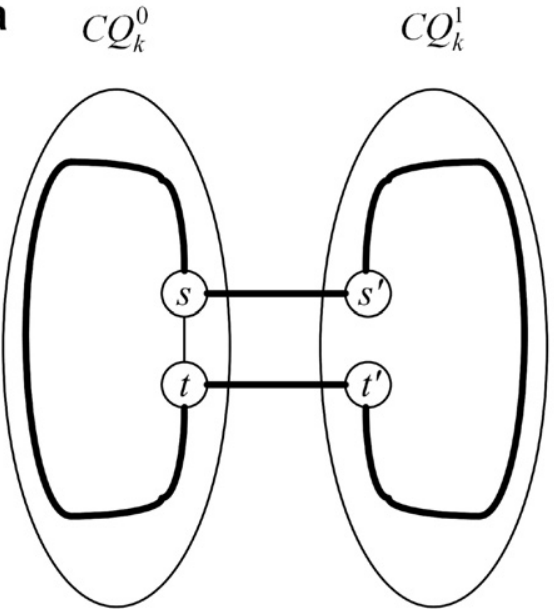

b $\quad C Q_{k}^{0} \quad C Q_{k}^{1}$

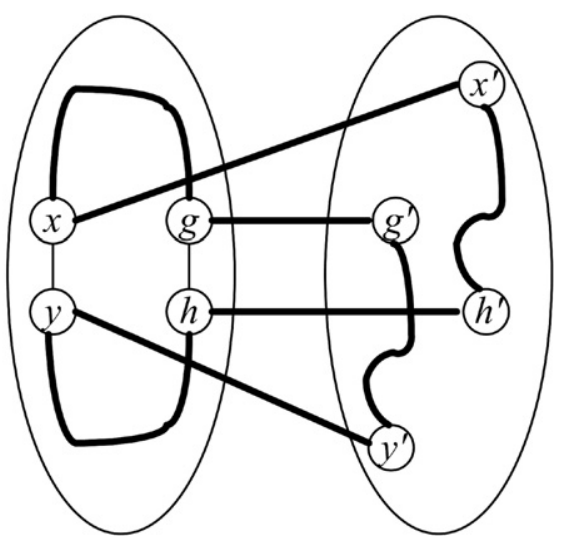

Fig. 6. Construction of a fault-free Hamiltonian cycle in $C Q_{k+1}$. (a) $f_{0} \leqslant 2 k-5$. (b) $f_{0}=2 k-3$. 
Case 2: $f_{0}=2 k-4$. We have $f_{1}+f_{c} \leqslant 1$. Similarly, there is at most one node in $C Q_{k}^{0}$ that is incident with only one fault-free link in $C Q_{k}^{0}$. If every node in $C Q_{k}^{0}$ is incident with at least two fault-free links in $C Q_{k}^{0}$, then we arbitrarily select a faulty link $(x, y)$ in $C Q_{k}^{0}$ with $\left(x, x^{\prime}\right)$ and $\left(y, y^{\prime}\right)$ fault-free, where $x^{\prime}=c_{k+1}(x)$ and $y^{\prime}=c_{k+1}(y)$. By the induction hypothesis (imagining that $(x, y)$ is fault-free), there exists a Hamiltonian cycle, denoted by $C$, in $C Q_{k}^{0}$ that can avoid all faulty links but $(x, y)$ in $C Q_{k}^{0}$. A desired fault-free Hamiltonian cycle can be obtained by the construction method of Fig. 6a (replacing $(s, t)$ with $(x, y)$ if $(x, y)$ is contained in $C$, and replacing $(s, t)$ with another link in $C$ if $(x, y)$ is not contained in $C)$. On the other hand, if there is a node $p$ in $C Q_{k}^{0}$ that is incident with only one fault-free link in $C Q_{k}^{0}$, then we arbitrarily select a faulty link $(p, q)$ in $C Q_{k}^{0}$ with $\left(q, q^{\prime}\right)$ fault-free, where $q^{\prime}=c_{k+1}(q)$. By the induction hypothesis (imaging that $(p, q)$ is fault-free), there is a Hamiltonian cycle that can avoid all faulty links but $(p, q)$ in $C Q_{k}^{0}$. A desired fault-free Hamiltonian cycle can be obtained by the construction method of Fig. 6a (replacing $(s, t)$ with $(p, q))$.

Case 3: $f_{0}=2 k-3$. We have $f_{1}=f_{c}=0$. There are at most two nodes in $C Q_{k}^{0}$ that are incident with only one fault-free link in $C Q_{k}^{0}$. We first assume that every node in $C Q_{k}^{0}$ is incident with at least two fault-free links in $C Q_{k}^{0}$. There are two faulty links $(x, y)$ and $(g, h)$ in $C Q_{k}^{0}$ so that we have $\{x, y\} \cap\{g, h\}=\varnothing$. By the induction hypothesis (imagining that $(x, y)$ and $(g, h)$ are fault-free), there exists a Hamiltonian cycle, denoted by $C^{\prime}$, in $C Q_{k}^{0}$ that can avoid all faulty links but $(x, y)$ and $(g, h)$.

If both $(x, y)$ and $(g, h)$ are contained in $C^{\prime}$, then a desired fault-free Hamiltonian cycle can be constructed as shown in Fig. 6b, where we have $x^{\prime}=c_{k+1}(x), y^{\prime}=c_{k+1}(y), g^{\prime}=c_{k+1}(g), h^{\prime}=c_{k+1}(h)$, and the two paths $P_{y^{\prime}, g^{\prime}}$ and $P_{x^{\prime}, h^{\prime}}$ with $V\left(P_{y^{\prime}, g^{\prime}}\right) \cap V\left(P_{x^{\prime}, h^{\prime}}\right)=\varnothing$ and $V\left(P_{y^{\prime}, g^{\prime}}\right) \cup V\left(P_{x^{\prime}, h^{\prime}}\right)=V\left(C Q_{k}^{0}\right)$ can be assured by Lemma 4. Otherwise, if $(x, y)$ or $(g, h)$ are not contained in $C^{\prime}$, then a desired fault-free Hamiltonian cycle can be obtained by the construction method of Fig. 6a (replacing $(s, t)$ with $(x, y)$ (or $(g, h))$ if $(x, y)$ (or $(g, h))$ is contained in $C^{\prime}$, and replacing $(s, t)$ with any link in $C^{\prime}$ if neither $(x, y)$ nor $(g, h)$ is contained in $\left.C^{\prime}\right)$.

Then we assume that there is exactly one node $p$ in $C Q_{k}^{0}$ that is incident with only one fault-free link in $C Q_{k}^{0}$. Suppose that $(p, q)$ is an arbitrary faulty link in $C Q_{k}^{0}$. There are at least $(2 k-3)-(k-3)-(k-1)=1$ faulty link in $C Q_{k}^{0}$ whose end nodes are neither $p$ nor $q$. Let $(x, y)$ be such a faulty link in $C Q_{k}^{0}$, i.e., $\{p, q\} \cap\{x, y\}=\varnothing$. By the induction hypothesis (imagining that $(p, q)$ and $(x, y)$ are fault-free), there exists a Hamiltonian cycle in $C Q_{k}^{0}$ that can avoid all faulty links but $(p, q)$ and $(x, y)$. Notice that $(p, q)$ should be included in the Hamiltonian cycle.

If $(x, y)$ is contained in the Hamiltonian cycle, then a desired fault-free Hamiltonian cycle can be obtained by the construction method of Fig. $6 \mathrm{~b}$ (replacing $(g, h)$ with $(p, q)$ ). Otherwise, a desired fault-free Hamiltonian cycle can be obtained by the construction method of Fig. 6a (replacing $(s, t)$ with $(p, q)$ ).

Next, we assume that there are two nodes $p$ and $w$ in $C Q_{k}^{0}$ that each are incident with only one fault-free link in $C Q_{k}^{0}$. Notice that $(p, w)$ is a faulty link, for otherwise there are more than $2 k-3$ faulty links in $C Q_{k}^{0}$, which is a contradiction. Suppose that $(p, q)$ and $(w, z)$ are two faulty links with $\{p, q\} \cap\{w, z\}=\varnothing$. By the induction hypothesis (imagining that $(p, q)$ and $(w, z)$ are fault-free), there exists a Hamiltonian cycle in $C Q_{k}^{0}$ that can avoid all faulty links but $(p, q)$ and $(w, z)$. Notice that $(p, q)$ and $(w, z)$ should be included

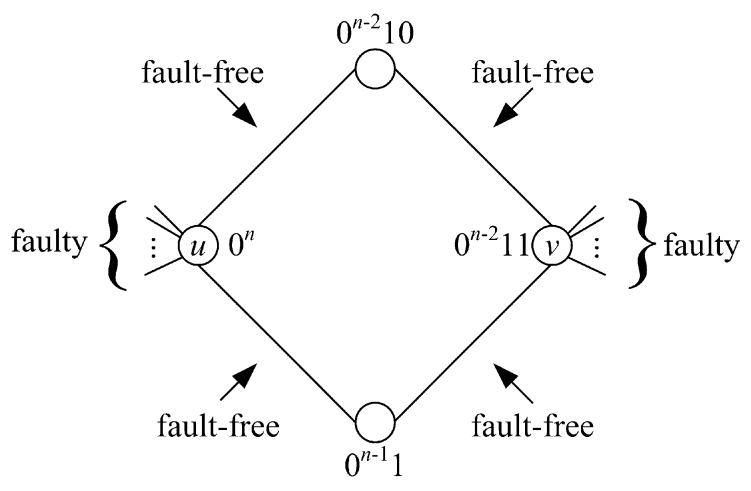

Fig. 7. A distribution of $2 n-4$ link faults in $C Q_{n}$. 
Table 1

Values of formula (1)

\begin{tabular}{lc}
\hline$n$ & Values of $(1)$ \\
\hline 5 & $>0.999400$ \\
6 & $>0.999995$ \\
7 & $>\left(1-8 \times 10^{-9}\right.$ \\
8 & $>(1-4) \times 10^{-12}$ \\
9 & $>(1-4) \times 10^{-16}$ \\
10 & $>(1-9) \times 10^{-21}$ \\
11 & $>(1-6) \times 10^{-26}$ \\
12 & $>(1-9) \times 10^{-32}$ \\
13 & $>(1-4) \times 10^{-38}$ \\
14 & $>(1-3) \times 10^{-45}$ \\
15 & $>(1-7) \times 10^{-53}$ \\
16 & $>(1-5) \times 10^{-61}$ \\
17 & $>(1-6) \times 10^{-70}$ \\
19 & $>(1-3) \times 10^{-79}$ \\
\hline
\end{tabular}

in the Hamiltonian cycle. A desired fault-free Hamiltonian cycle can be obtained by the construction method of Fig. $6 \mathrm{~b}$ (replacing $(x, y)$ and $(g, h)$ with $(p, q)$ and $(w, z)$, respectively).

Finally, we show a distribution of $2 n-4$ link faults over a $C Q_{n}$ such that no fault-free Hamiltonian cycle can be found in the faulty $C Q_{n}$. Let us consider two nodes $u=0^{n}$ ( $n$ consecutive 0 's) and $v=0^{n-2} 1^{2}$ of the $C Q_{n}$. Refer to Fig. 7 where $u$ and $v$ have their 0-links and 1-links fault-free and the other $n-2$ links faulty. It is easy to see that no fault-free Hamiltonian cycle exists in the faulty $C Q_{n}$.

\section{The probability}

Recall that we have assumed in Theorem 1 that each node in $C Q_{n}$ is incident with at least two fault-free links, while there are up to $2 n-5$ link faults in the $C Q_{n}$, where $n \geqslant 3$. In this section, we further show that the assumption is practically meaningful by analyzing its probability when the $C Q_{n}$ contains $2 n-5$ link faults. By $p_{n}$ we denote the probability that each node in such a faulty $C Q_{n}$ is incident with at least two fault-free links. Since there are a total of $n \times 2^{n-1}$ links contained in $C Q_{n}$, there are $\left(\begin{array}{c}n \times 2^{n-1} \\ 2 n-5\end{array}\right)$ ways to distribute the $2 n-5$ link faults. All these fault distributions are assumed having equal probability of occurrence.

Clearly, $p_{3}=1$ and $p_{4}$ is computed as $1-\frac{16 \times\left(\begin{array}{l}4 \\ 3\end{array}\right)}{\left(\begin{array}{c}32 \\ 3\end{array}\right)}=0.987097$, where $16 \times\left(\begin{array}{l}4 \\ 3\end{array}\right)$ is the number of fault distributions having some node incident with three link faults. When $n \geqslant 5$, there are $2^{n} \times$ $\left(\begin{array}{c}n \times 2^{n-1}-n \\ n-5\end{array}\right)\left(\right.$ or $\left.2^{n} \times\left(\begin{array}{c}n \\ n-1\end{array}\right) \times\left(\begin{array}{c}n \times 2^{n-1}-n \\ n-4\end{array}\right)\right)$ fault distributions having some node incident with $n$ (or $n-1)$ link faults. It is not difficult to check that $\left(\begin{array}{c}n \times 2^{n-1}-n \\ n-4\end{array}\right)>\left(\begin{array}{c}n \times 2^{n-1}-n \\ n-5\end{array}\right)$ for $n \geqslant 5$. Thus, we have

$$
p_{n}=1-\frac{2^{n} \times\left(\begin{array}{c}
n \times 2^{n-1}-n \\
n-5
\end{array}\right)+2^{n} \times\left(\begin{array}{c}
n \\
n-1
\end{array}\right) \times\left(\begin{array}{c}
n \times 2^{n-1}-n \\
n-4
\end{array}\right)}{\left(\begin{array}{c}
n \times 2^{n-1} \\
2 n-5
\end{array}\right)}
$$




$$
\begin{aligned}
& >1-\frac{2^{n} \times(n+1) \times\left(\begin{array}{c}
n \times 2^{n-1}-n \\
n-4
\end{array}\right)}{\left(\begin{array}{c}
n \times 2^{n-1} \\
2 n-5
\end{array}\right)} \\
& =1-2^{n} \times(n+1) \times\left(n \times 2^{n-1}-(2 n-5)\right) \times \frac{\prod_{i=n-3}^{2 n-5} i}{\prod_{i=0}^{n-1}\left(n \times 2^{n-1}-i\right)} .
\end{aligned}
$$

Table 1 shows the values of formula (1) for $n=5,6, \ldots, 20$. When $n$ increases, the values of (1) go up rapidly and toward 1. Consequently, the probability that each node of $C Q_{n}$ with at most $2 n-5$ link faults is incident with at least two fault-free links is very close to 1 , even if $n$ is small.

\section{Discussion and conclusion}

In this paper, with the assumption of at least two fault-free links incident to each node, we have shown that there exists a fault-free Hamiltonian cycle in an $n$-dimensional crossed cube $\left(C Q_{n}\right)$ with up to $2 n-5$ link faults. We also verified that the assumption is practically meaningful by evaluating its occurrence probability, which is very close to 1 , even if $n$ is small. Besides, the result is optimal with respect to the number of link faults tolerated. A recursive algorithm for constructing a fault-free Hamiltonian cycle can be easily obtained from the proof of Theorem 1 .

With the same assumption, a previous work by Chan and Lee [2] constructed a fault-free Hamiltonian cycle in an $n$-dimensional hypercube with $2 n-5$ link faults. The hypercube is highly symmetric, and it can be partitioned at any dimension into two smaller hypercubes. With this favorable property, fault-free Hamiltonian cycles were successfully obtained for some intractable distributions of link faults. On the other hand, the crossed cube is not symmetric and it can be partitioned into two smaller crossed cubes at only two dimensions. It was the major difficulty encountered when we tried to extend the result of [2] to the crossed cube. In order to achieve the extension, some new properties, as described in Section 3, on the crossed cube were therefore derived.

It was shown in [22] that each member in the class of hypercube-like networks [27] contains a Hamiltonian cycle. Both the hypercube and crossed cube belong to the class. With the same assumption, the hypercube can tolerate up to $2 n-5$ link faults, while retaining a fault-free Hamiltonian cycle. We are wondering how many link faults the other hypercube-like networks can tolerate, while retaining a fault-free Hamiltonian cycle.

Moreover, it was shown in [29] that a $C Q_{n}$ with at most $n-3$ (link or node) faults contains fault-free cycles of every possible length, except three. In [18], the authors showed, with the same assumption, that a $C Q_{n}$ with at most $2 n-5$ link faults contains fault-free cycles of lengths ranging from 4 to $2^{n}$. We are interested in exploring other topological properties, such as connectivity, diameter and Hamiltonian-connectedness, of the crossed cube under the assumption of conditional faults.

In [14], the h-extraconnectivity (h-edge-extraconnectivity) problem was defined for a graph $G$, which, assuming that $S$ was a set of faulty vertices (edges) in $G$, was required to compute the minimum $|S|$ so that $G-S$ was disconnected and every component of $G-S$ had at least $h+1$ nodes, where $h \geqslant 0$ was an integer. When $h=0$, the $h$-extraconnectivity ( $h$-edge-extraconnectivity) of $G$ is equal to the vertex (edge) connectivity of $G$. When $h=1$, the $h$-extraconnectivity ( $h$-edge-extraconnectivity) of $G$ is equal to the conditional vertex (edge) connectivity $[9,10]$ of $G$. Recently, the two problems were further solved in [31,32] for $h=2$. In [31], the 2-edge-extraconnectivities of hypercubes, twisted cubes, crossed cubes and Möbius cubes were computed. In [32], the 2-extraconnectivity and 2-edge-extraconnectivity of folded hypercubes were computed. We are also interested in studying the two problems on hypercube-like networks.

\section{Acknowledgements}

The authors would like to express their gratitude to the anonymous reviewers for their valuable comments and suggestions which improve the paper a lot. The authors are also grateful to the National Science Council of the Republic of China, Taiwan for financially supporting this research under Contract No. NSC 94-2213-E239-014. 


\section{References}

[1] Y.A. Ashir, I.A. Stewart, Fault-tolerant embedding of Hamiltonian circuits in $k$-ary $n$-cube, SIAM Journal on Discrete Mathematics 15 (3) (2002) 317-328.

[2] M.Y. Chan, S.J. Lee, On the existence of Hamiltonian circuits in faulty hypercubes, SIAM Journal on Discrete Mathematics 4 (4) (1991) 511-527.

[3] C.P. Chang, T.Y. Sung, L.H. Hsu, Edge congestion and topological properties of crossed cube, IEEE Transactions on Parallel and Distributed Systems 11 (1) (2000) 64-80.

[4] G.Y. Chang, G.H. Chen, G. J Chang, $(t, k)$-Diagnosis for matching composition networks, IEEE Transactions on Computers 55 (1) (2006) 88-92.

[5] K. Day, The conditional node connectivity of the $k$-ary $n$-cube, Journal of Interconnection Networks 5 (1) (2004) $13-26$.

[6] K. Efe, A variation on the hypercube with lower diameter, IEEE Transactions on Computers 40 (11) (1991) $1312-1316$.

[7] K. Efe, The crossed cube architecture for parallel computation, IEEE Transactions on Parallel and Distributed Systems 3 (5) (1992) $513-524$.

[8] K. Efe, P.K. Blackwell, W. Slough, T. Shiau, Topological properties of the crossed cube architecture, Parallel Computing 20 (1994) 1763-1775.

[9] A.H. Esfahanian, Generalized measures of fault-tolerance with application to $n$-cube networks, IEEE Transactions on Computers 38 (11) (1989) 1586-1591.

[10] A.H. Esfahanian, S.L. Hakimi, On computing a conditional edge-connectivity of a graph, Information Processing Letters 27 (1988) 195-199.

[11] J. Fan, X. Jia, X. Lin, Complete path embedding in crossed cubes, Information Sciences 176 (22) (2005) $3332-3346$.

[12] J. Fan, X. Lin, X. Jia, Optimal path embedding in crossed cube, IEEE Transactions on Parallel and Distributed Systems 16 (12) (2005) 1190-1200.

[13] J. Fan, X. Lin, The $t / k$-diagnosability of the BC graphs, IEEE Transactions on Computers 54 (2) (2005) $176-184$.

[14] J. Fäbrega, M.A. Fiol, Extraconnectivity of graphs with large girth, Discrete Mathematics 127 (1994) 163-170.

[15] J.S. Fu, Longest fault-free paths in hypercubes with vertex faults, Information Sciences 176 (7) (2006) 759-771.

[16] F. Harary, Conditional connectivity, Networks 13 (1983) 347-357.

[17] W.T. Huang, Y.C. Chuang, L.H. Hsu, J.M. Tan, On the fault-tolerant hamiltonicity of crossed cubes, IEICE Transactions on Fundamentals E85-A (6) (2002) 1359-1371.

[18] H.S. Hung, J.S. Fu, G.H. Chen, Embedding fault-free cycles in crossed cubes with conditional link faults, in preparation.

[19] P. Kulasinghe, S. Betayeb, Embedding binary trees into crossed cubes, IEEE Transactions on Computers 44 (7) (1995) 923-929.

[20] P. Kulasinghe, Connectivity of the crossed cube, Information Processing Letters 61 (1997) 221-226.

[21] S. Latifi, Combinatorial analysis of the fault-diameter of the $n$-cube, IEEE Transactions on Computers 42 (1) (1993) $27-33$.

[22] C.D. Park, K.Y. Chwa, Hamiltonian properties on the class of hypercube-like networks, Information Processing Letters 91 (2004) 11-17.

[23] L. Qiao, Z. Yi, Restricted connectivity and restricted fault diameter of some interconnection networks, DIMACS Series in Discrete Mathematics and Theoretical Computer Science 21 (1995).

[24] Y. Rouskov, S. Latifi, P.K. Srimani, Conditional fault diameter of star graph networks, Journal of Parallel and Distributed Computing 33 (1996) 91-97.

[25] Y. Saad, M.H. Schultz, Topological properties of hypercubes, IEEE Transactions on Computers 37 (7) (1988) $867-872$.

[26] C.H. Tsai, Linear array and ring embeddings in conditional faulty hypercubes, Theoretical Computer Science 314 (2004) $431-443$.

[27] A.S. Vaidya, P.S.N. Rao, S.R. Shankar, A class of hypercube-like networks, in: Proceedings of the Fifth IEEE Symposium on Parallel and Distributed Processing, Los Alamitos, CA, 1993, pp. 800-803.

[28] A.Y. Wu, Embedding of tree networks into hypercubes, Journal of Parallel and Distributed Computing 2 (3) (1985) $23-249$.

[29] M.C. Yang, T.K. Li, J.M. Tan, L.H. Hsu, Fault-tolerant cycle-embedding of crossed cubes, Information Processing Letters 88 (2003) $149-154$.

[30] M.C. Yang, T.K. Li, J.M. Tan, L.H. Hsu, On embedding cycles into faulty twisted cubes, Information Sciences 176 (6) (2006) 676690.

[31] Q. Zhu, J.M. Xu, M. Lü, Edge fault tolerance analysis of a class of networks, Applied Mathematics and Computation 172 (1) (2006) $111-121$.

[32] Q. Zhu, J.M. Xu, X. Hou, M. Xu, On reliability of the folded hypercubes, Information Sciences 177 (8) (2007) $1782-1788$.

[33] http://inrg.csie.ntu.edu.tw/CQ_Hamiltonian/index.htm. 